\title{
SÍNTESE DE NOVAS FITOTOXINAS DERIVADAS DO 8-OXABICICLO[3.2.1]OCT-6-EN-3-ONA
}

\author{
Luiz C. A. Barbosa*, Célia R. A. Maltha, Antônio J. Demuner e Flávia R. Ganen \\ Departamento de Química, Universidade Federal de Viçosa, 36571-000 Viçosa - MG \\ Antônio A. da Silva \\ Departamento de Fitotecnia, Universidade Federal de Viçosa, 36571-000 Viçosa - MG
}

Recebido em 27/4/04; aceito em 22/9/04; publicado na web em 2/2/05

\begin{abstract}
SYNTHESIS OF NEW PHYTOTOXINS DERIVED FROM 8-OXABICYCLO[3.2.1]OCT-6-EN-3-ONE. The [4+3] cycloaddition was utilized in order to prepare 8-oxabicyclo[3.2.1] oct-6-en-3-one (1) derivatives. The correspondent acetonide 6 was converted into several alcohols (11-16). Addition of aryllithium reagents to 6 resulted in 3-(2-fluorophenyl)-6,7-exo-isopropylidenedioxy8-oxabicyclo[3.2.1] octan-3 $\alpha$-ol $(\mathbf{1 1}, 72 \%)$ and 3-(2,4-dimethoxyphenyl)-6,7-exo-isopropylidenedioxy-8-oxabicyclo[3.2.1]octan$3 \alpha$-ol $(\mathbf{1 6}, 20 \%)$. The 3-butyl-6,7-exo-isopropylidenedioxy-8-oxabicyclo[3.2.1] octan-3 $\alpha$-ol $(\mathbf{1 5}, 56 \%)$ was obtained through a

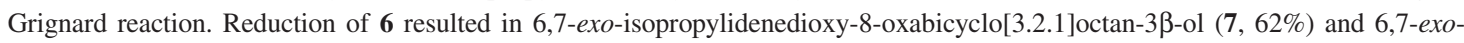
isopropylidenedioxy-8-oxabicyclo[3.2.1] octan-3 $\alpha$-ol $(\mathbf{8}, 20 \%)$. The alcohols were treated with thionyl chloride in pyridine, and the corresponding alkenes were obtained with $31-80 \%$ yield. The effect of these compounds on the development of radicle and aerial parts of Sorghum bicolor was evaluated.
\end{abstract}

Keywords: [4+3] cycloaddition; oxyallyl cations; herbicides.

\section{INTRODUÇÃO}

A utilização de herbicidas é a principal estratégia utilizada para o controle de plantas daninhas na agricultura empresarial. Esses produtos são largamente utilizados devido à alta eficiência observada, fácil utilização, ação rápida e ao custo bastante acessível. Todavia o uso repetido de um mesmo herbicida exerce elevada pressão de seleção sobre a população de plantas daninhas, o que promove a seleção de biótipos resistentes a esses produtos ${ }^{1}$.

O potencial biológico de uma classe de compostos está intimamente relacionado com a natureza dos grupos funcionais e/ou substituintes presentes na molécula. Nesse sentido, a indústria agroquímica está constantemente desenvolvendo novas metodologias de síntese orgânica com o objetivo de disponibilizar no mercado novos compostos cada vez mais seletivos, eficientes e seguros sob o ponto de vista ambiental ${ }^{2}$. Por exemplo, o composto imazapir é um herbicida não seletivo enquanto o imazetapir (Figura 1), pertencente à mesma classe do imazapir e que difere apenas pela presença de um grupo etil no anel piridínico ${ }^{3}$, é seletivo para a cultura da soja ${ }^{4}$. Além dessas características, o imazapir apresenta persistência no solo muito superior ao imazetapir podendo, assim, causar maior impacto ambiental $^{4}$. Essa diferença de interação entre as moléculas e o ambiente devido a pequenas modificações estruturais, juntamente com a diferença observada na atividade herbicida, reflete a necessidade de se investir nos processos de modificação molecular a partir de com-
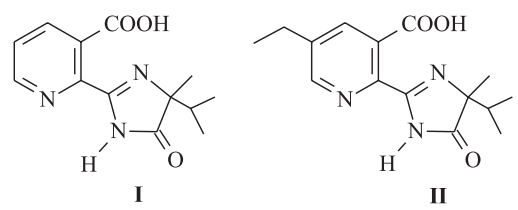

Figura 1. Estruturas do imazapir (I) e imazetapir (II)

\footnotetext{
*e-mail: lcab@ufv.br
}

postos de ação conhecida para a busca de novos compostos com elevado poder comercial.

Bioensaios realizados utilizando diversas espécies de culturas e de plantas daninhas como plantas indicadoras evidenciaram o potencial herbicida de derivados do 8-oxabiciclo[3.2.1]oct-6-en-3-ona (1) ${ }^{5-11}$, obtido pela reação de cicloadição [4+3] entre cátions oxialílicos e dienos (Figura 2) ${ }^{12}$.

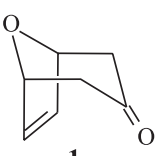

1

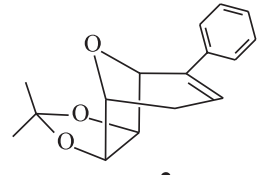

2

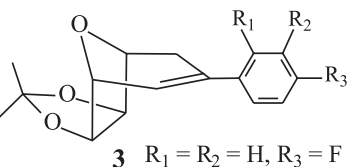

$4 \mathrm{R}_{1}=\mathrm{R}_{2}=\mathrm{R}_{3}=\mathrm{H}$

$5 \quad \mathrm{R}_{1}=\mathrm{R}_{3}=\mathrm{H}, \mathrm{R}_{2}=\mathrm{CH}_{3}$
Figura 2. Derivados do 8-oxabiciclo[3.2.1]oct-6-en-3-ona (1)

Em um dos bioensaios realizados observou-se que a inibição de crescimento sobre o sistema radicular de Sorghum bicolor L. causada pelo composto 2 , na concentração de $250 \mu \mathrm{g} \mathrm{mL} \mathrm{m}^{-1}$, foi de $83 \%$. Para os compostos 3, 4 e 5, na concentração de $6,6 \mu \mathrm{g} \mathrm{g}^{-1}$, também foi observada inibição sobre o crescimento radicular de S. bicolor $(54,4,59,4$ e 66,3\%, respectivamente $)^{10}$. Resultados surpreendentes foram ainda obtidos com os compostos 3, 4 e 5 que, na concentração de $6,6 \mu \mathrm{g} \mathrm{g}$, causaram $100 \%$ de mortalidade em plantas de Cucumis sativus $\mathrm{L}^{10}$.

Diversos trabalhos de síntese abordando uma mesma metodologia sintética são descritos seqüencialmente na literatura para a obtenção de compostos análogos a um protótipo de ação conhecida. Estes trabalhos apresentam o preparo de compostos com variação de grupos funcionais e/ou substituintes, o que permite estabelecer uma correlação estrutura-atividade biológica ${ }^{13-15}$. Considerando-se o potencial herbicida de alquenos derivados do 8-oxabiciclo[3.2.1]oct-6-en-3ona $(\mathbf{1})^{10}$ e a eficácia da estratégia de "screening" aleatório na descoberta de novos compostos com atividade fitotóxica ${ }^{3}$, apresentamos nesse trabalho a síntese de novos derivados (7 a 11 e 15 a 19) do oxabiciclo 1 com o objetivo de avaliar a atividade fitotóxica dos mes- 
mos e dar continuidadade ao estudo iniciado anteriormente ${ }^{10}$, visando a descoberta de novas moléculas com potencial herbicida que possam ser incorporadas ao processo produtivo.

\section{PARTE EXPERIMENTAL}

\section{Procedimentos experimentais gerais}

As temperaturas de fusão foram determinadas em aparelho Kofler $\mathrm{R}$ Winkel Sotting e corrigidas. Os espectros na região do infravermelho obtidos em espectrômetro Perkin Elmer FTIR 1000, na região de 4000 a $600 \mathrm{~cm}^{-1}$, foram registrados utilizando-se pastilhas de $\mathrm{KBr}$ para os compostos sólidos, ou como filme líquido no caso de amostras oleosas. Os espectros de RMN ${ }^{1} \mathrm{H}$ e RMN ${ }^{13} \mathrm{C}$ foram obtidos em espectrômetro Bruker DRX 400, utilizando-se $\mathrm{CDCl}_{3}$ como solvente e TMS como referência interna. Os espectros de massas foram obtidos em espectrômetro V. G. Analytical ZAB-IF.

Os reagentes utilizados nas reações e os solventes utilizados para cromatografia em coluna de sílica gel foram previamente purificados e secados de acordo com os procedimentos descritos na literatu$\mathrm{ra}^{16}$. Todas as reações foram monitoradas por cromatografia em camada delgada utilizando-se placas de sílica gel Camlab-Polygram SILK/UV ${ }_{254}, \operatorname{com} 0,25 \mathrm{~mm}$ de espessura. Para a purificação dos compostos utilizou-se a cromatografia em coluna de sílica gel 60 (70230 mesh-ASTM, Merck).

O composto 1 foi sintetizado conforme procedimento descrito na literatura ${ }^{5}$. Os compostos $6,12,13$ e 14 foram novamente preparados, visando a avaliação da fitotoxicidade. Os dados físicos e espectroscópicos de 6, 12, 13 e $\mathbf{1 4}$ foram descritos anteriormente ${ }^{10}$.

\section{Procedimentos sintéticos}

6,7-exo-isopropilidenodioxi-8-oxabiciclo[3.2.1]octan-3 $\beta$-ol (7) e 6,7exo-isopropilidenodioxi-8-oxabiciclo[3.2.1]octan-3 $\alpha$-ol (8). A uma solução do acetonídeo 6 (0,495 g, 2,5 mmol), em metanol anidro (30 $\mathrm{mL})$ sob agitação magnética, adicionou-se boroidreto de sódio $(0,168$ g, 4,44 mmol). A mistura foi mantida sob agitação magnética por $5 \mathrm{~h}$, e aquecida a $40{ }^{\circ} \mathrm{C}$ durante $2 \mathrm{~h}$. Em seguida, foram adicionadas dez gotas de água gelada, sob agitação magnética, e a mistura foi concentrada em evaporador rotatório. $\mathrm{O}$ fracionamento do sólido obtido, por cromatografia em coluna de sílica-gel (hexano/Et ${ }_{2} \mathrm{O} 1: 4$ ), forneceu os álcoois isoméricos $7(0,31 \mathrm{~g}, 1,55 \mathrm{mmol}, 62 \%)$ e $8(0,1 \mathrm{~g}, 0,5 \mathrm{mmol}$, 20\%). Dados para 7: $\mathrm{T}_{\mathrm{f}}=156-157,3{ }^{\circ} \mathrm{C}$. IV $\left(\mathrm{KBr}, \mathrm{cm}^{-1}\right) \overline{\mathrm{v}}_{\text {max }}: 3350$, 3000, 2900, 1470, 1370, 1250, 1150, 1075, 1000, 860, 725, 650. RMN ${ }^{1} \mathbf{H}\left(400 \mathrm{MHz}, \mathrm{CDCl}_{3}\right) \delta: 1,31$ (s, Me); 1,48 (s, Me); 1,61 (ddd, $J_{2 \beta, 2 \alpha}=$ $J_{4 \beta, 4 \alpha}=12,8 \mathrm{~Hz}, J_{2 \beta, 3}=J_{4 \beta, 3}=11,9 \mathrm{~Hz}, J_{2 \beta, 1}=J_{4 \beta, 1}=4,1 \mathrm{~Hz}, \mathrm{H}-2 \beta, \mathrm{H}-$ $4 \beta$ ); 1,69 (s, OH); 1,96 (dd, $J_{2 \alpha, 2 \beta}=J_{4 \alpha, 4 \beta}=12,8 \mathrm{~Hz}, J_{2 \alpha, 3}=J_{4 \alpha, 3}=5,7$ $\mathrm{Hz}, \mathrm{H}-2 \alpha, \mathrm{H}-4 \alpha$ ); 3,63 (m, H-3); 4,29 (dd, $J_{1} \cong 2,9 \mathrm{~Hz}, J_{2} \cong 2,3 \mathrm{~Hz}, \mathrm{H}-$ 1, H-5); 4,51 (s, H-6, H-7). RMN ${ }^{13} \mathbf{C}\left(100 \mathrm{MHz}, \mathrm{CDCl}_{3}\right): 111,66$ $\left(\mathrm{CMe}_{2}\right) ; 83,06$ (C-6, C-7); 79,31 (C-1, C-5); 62,99 (C-3); 37,41 (C-2, C-4); 25,99 (Me); 24,47 (Me). EM, $\boldsymbol{m} / \boldsymbol{z}(\%): 185,00\left(\left[\mathrm{C}_{9} \mathrm{H}_{13} \mathrm{O}_{4}\right]^{+^{*}}, \mathrm{M}-\right.$ 15, 15); 107,00 (5); 97,00 (10), 83,00 (10); 79,00 (10); 59,00 (20); 43,00 (100). Dados para 8: $\mathrm{T}_{\mathrm{f}}=142-143,7^{\circ} \mathrm{C}$. IV $\left(\mathrm{KBr}, \mathrm{cm}^{-1}\right) \overline{\mathrm{v}}_{\max }$ : 3450, 3000, 2900, 1460, 1420, 1375, 1260, 1100, 950, 850, 700. RMN ${ }^{1} \mathbf{H}\left(400 \mathrm{MHz}, \mathrm{CDCl}_{3}\right.$ ) $\delta: 1,34$ (s, Me); 1,49 (s, Me); 1,52 (s, OH); 1,67 (ddd, $J_{2 \beta, 2 \alpha}=J_{4 \beta, 4 \alpha}=15,1 \mathrm{~Hz}, J_{2 \beta, 3}=J_{4 \beta, 3}=1,3 \mathrm{~Hz}, J_{2 \beta, 1}=J_{4 \beta, 5}=0,9 \mathrm{~Hz}$, $\mathrm{H}-2 \beta, \mathrm{H}-4 \beta$ ); 2,09 (dt, $J_{2 \alpha, 2 \beta}=J_{4 \alpha, 4 \beta}=15,1 \mathrm{~Hz}, J_{2 \alpha, 1}=J_{4 \alpha, 5} \cong J_{2 \alpha, 3}=J_{4 \alpha, 3}$ $=4,7 \mathrm{~Hz}, \mathrm{H}-2 \alpha, \mathrm{H}-4 \alpha) ; 4,10(\mathrm{~m}, \mathrm{H}-3) ; 4,21\left(\mathrm{dd}, J_{1,2 \mathrm{a}}=J_{5,4 \mathrm{a}}=4,7 \mathrm{~Hz}\right.$, $\left.J_{1,2 \mathrm{~b}}=J_{5,4 \mathrm{~b}}=0,9 \mathrm{~Hz}, \mathrm{H}-1, \mathrm{H}-5\right) ; 5,05$ (s, H-6, H-7). RMN ${ }^{13} \mathbf{C}(100$ $\left.\mathrm{MHz}, \mathrm{CDCl}_{3}\right): 111,33\left(\mathrm{CMe}_{2}\right) ; 83,73$ (C-6, C-7); 79,22 (C-1, C-5); 63,61 (C-3); 35,49 (C-2, C-4); 26,13 (Me); 24,57 (Me). EM, m/z (\%): $185,00\left(\left[\mathrm{C}_{9} \mathrm{H}_{13} \mathrm{O}_{4}\right]^{+*}, \mathrm{M}-15,12\right) ; 107,00$ (5); 97,00 (10), 83,00 (10); 79,00 (10); 59,00 (20); 43,00 (100).
3 $\alpha$-cloro-6,7-exo-isopropilidenodioxi-8-oxabiciclo[3.2.1]octano (9)

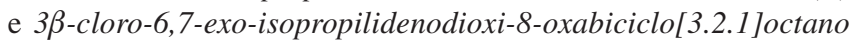
(10): A uma mistura dos álcoois 7 e $8(0,10 \mathrm{~g} ; 0,5 \mathrm{mmol})$ em piridina anidra (4 mL, 4,97 mmol) foi adicionado cloreto de tionila $(2 \mathrm{~mL}$, $27,4 \mathrm{mmol}$ ). A mistura resultante foi mantida sob agitação magnética durante $7 \mathrm{~h}$ e, em seguida, resfriada em banho de gelo para adição de gotas de solução de $\mathrm{HCl}\left(2 \mathrm{~mol} \mathrm{~L}^{-1}\right)$ até a completa neutralização do excesso de piridina. O produto foi extraído com diclorometano (3 x $20 \mathrm{~mL}$ ) e a fase orgânica foi lavada com solução saturada de $\mathrm{NaCl}$, secada sobre $\mathrm{MgSO}_{4}$ anidro e concentrada em evaporador rotatório. A purificação do material obtido, por cromatografia em coluna de sílica-gel (hexano/Et $\mathrm{O}_{2}$ 4,5:1), forneceu os cloretos $9(0,034 \mathrm{~g}$, $0,16 \mathrm{mmol}, 32 \%)$ e $\mathbf{1 0}(0,003 \mathrm{~g}, 0,01 \mathrm{mmol}, 2 \%)$. Dados para 9: $\mathrm{T}_{\mathrm{f}}=$ $104-105,5^{\circ} \mathrm{C} . \mathbf{I V}\left(\mathrm{KBr}, \mathrm{cm}^{-1}\right) \overline{\mathrm{v}}_{\max }: 2960,2900,1650,1450,1400$, $1250,1100,1050,850 . \mathbf{R M N}^{1} \mathbf{H}\left(400 \mathrm{MHz}^{\max } \mathrm{CDCl}_{3}\right) \delta: 1,36(\mathrm{~s}, \mathrm{Me})$; $1,49(\mathrm{~s}, \mathrm{Me}) ; 2,03\left(\mathrm{dd}, J_{2 \beta, 2 \alpha}=J_{4 \beta, 4 \alpha}=16,0 \mathrm{~Hz}, J_{2 \beta, 3}=J_{4 \beta, 3}=0,9 \mathrm{~Hz}\right.$, $\mathrm{H}-2 \beta, \mathrm{H}-4 \beta$ ); 2,40 (ddd, $J_{2 \alpha, 2 \beta}=J_{4 \alpha, 4 \beta}=16,0 \mathrm{~Hz}, J_{2 \alpha, 3}=J_{4 \alpha, 3}=6,0 \mathrm{~Hz}$, $\left.J_{2 \alpha, 1}=J_{4 \alpha, 5}=4,6 \mathrm{~Hz}, \mathrm{H}-2 \alpha, \mathrm{H}-4 \alpha\right) ; 4,27\left(\mathrm{dd}, J_{1,2 \alpha}=J_{5,4 \alpha}=4,6 \mathrm{~Hz}, J_{1,2 \beta}\right.$ $\left.=J_{5,4 \mathrm{~b}}=0,8 \mathrm{~Hz}, \mathrm{H}-1, \mathrm{H}-5\right) ; 4,40\left(2 \mathrm{t}, J_{3,2 \alpha}=J_{3,4 \alpha}=6,0 \mathrm{~Hz}, J_{3,2 \beta}=J_{3,4 \beta}\right.$ $=0,9 \mathrm{~Hz}, \mathrm{H}-3) ; 5,00$ (s, H-6, H-7). RMN ${ }^{13} \mathbf{C}\left(100 \mathrm{MHz}, \mathrm{CDCl}_{3}\right)$ : 111,32 $\left(\mathrm{CMe}_{2}\right)$; 83,02 (C-6, C-7); 79,30 (C-1, C-5); 52,35 (C-3); 35,92 (C-2, C-4); 26,06 (Me); 24,56 (Me). EM, $\boldsymbol{m} / z$ (\%): 218,00 $\left(\mathrm{M}^{+?}, 85\right) ; 203,00$ (100); 183,00 (50), 167,00 (35); 131,00 (27); 125,00 (40); 95,00 (23). Dados para 10: $\mathrm{T}_{\mathrm{f}}=88-89,8{ }^{\circ} \mathrm{C}$. IV $\left(\mathrm{KBr}, \mathrm{cm}^{-1}\right)$ $\bar{v}_{\max }: 2960,2900,1650,1450,1400,1250,1100,1050,850 . \mathbf{R M N}$ ${ }^{1} \mathbf{H}\left(400 \mathrm{MHz}_{\mathrm{maCl}}\right) \delta: 1,26(\mathrm{~s}, \mathrm{Me}) ; 1,32(\mathrm{~s}, \mathrm{Me}) ; 2,01$ (ddd, $J_{2 \beta, 2 \alpha}$ $\left.=J_{4 \beta, 4 \alpha}=13,0 \mathrm{~Hz}, J_{2} \cong 13,0 \mathrm{~Hz}, J_{3}=4,0 \mathrm{~Hz}, \mathrm{H}-2 \beta, \mathrm{H}-4 \beta\right) ; 2,10$ (dd, $\left.J_{2 \alpha, 2 \beta}=J_{4 \alpha, 4 \beta}=13,0 \mathrm{~Hz}, J_{2}=5,8 \mathrm{~Hz}, \mathrm{H}-2 \alpha, \mathrm{H}-4 \alpha\right) ; 3,81$ (m, H-3); 4,28 (m, H-1, H-5); 4,55 (s, H-6, H-7). RMN ${ }^{13} \mathbf{C}\left(100 \mathrm{MHz}, \mathrm{CDCl}_{3}\right.$ ): 111,30 $\left(\mathrm{CMe}_{2}\right) ; 82,79$ (C-6, C-7); 80,02 (C-1, C-5); 50,63 (C-3); 38,67 (C-2, C-4); 25,95 (Me); 24,48 (Me).

3-(2-fluorofenil)-6,7-exo-isopropilidenodioxi-8-oxabiciclo[3.2.1]octan-3 $\alpha$-ol (11): A uma solução de 1-bromo-2fluorobenzeno $(0,441 \mathrm{~g}, 2,52 \mathrm{mmol})$, em THF anidro $(5 \mathrm{~mL})$, foram adicionados $2 \mathrm{~mL}$ de solução de butil lítio (1,6 mol L-1 em hexano, $3,2 \mathrm{mmol})$ a $-78^{\circ} \mathrm{C}$. Após $1,5 \mathrm{~h}$, adicionou-se a solução do acetonídeo 6 (0,2 g, 1,01 mmol, $3 \mathrm{~mL}$ de THF anidro). A mistura foi mantida a $-78{ }^{\circ} \mathrm{C}$ durante $1 \mathrm{~h}$ e, em seguida, à temperatura ambiente por $3 \mathrm{~h}$. Foram adicionados $10 \mathrm{~mL}$ de água e o produto foi extraído com diclorometano (3 x $20 \mathrm{~mL}$ ). A fase orgânica foi lavada com solução saturada de $\mathrm{NaCl}(20 \mathrm{~mL})$, secada sobre $\mathrm{MgSO}_{4}$ anidro e concentrada em evaporador rotatório. A purificação do sólido obtido, por cromatografia em coluna de sílica-gel (hexano/Et $\mathrm{O}_{2} 1: 1$ ), forneceu o álcool $11(0,211 \mathrm{~g}, 0,72 \mathrm{mmol}, 72 \%) . \mathrm{T}_{\mathrm{f}}=169-170^{\circ} \mathrm{C}$. IV $\left(\mathrm{KBr}, \mathrm{cm}^{-1}\right)$ $\bar{v}_{\max }: 3420,3000,2900,1625,1500,1450,1350,1225,1100,900$, 750. $\mathbf{R M N}{ }^{1} \mathbf{H}\left(400 \mathrm{MHz}, \mathrm{CDCl}_{3}\right.$ ) $\delta: 1,37$ (s, Me); 1,51 (s, Me); 1,92 $\left(\mathrm{d}, J_{2 \alpha, 2 \beta}=J_{4 \alpha, 4 \beta}=14,8 \mathrm{~Hz}, \mathrm{H}-2 \alpha, \mathrm{H}-4 \alpha\right) ; 2,05(\mathrm{~s}, \mathrm{OH}) ; 2,54$ (dd, $\left.J_{2 \beta, 2 \alpha}=J_{4 \beta, 4 \alpha}=14,8 \mathrm{~Hz}, J_{2 \beta, 1}=J_{4 \beta, 5}=4,7 \mathrm{~Hz}, \mathrm{H}-2 \beta, \mathrm{H}-4 \beta\right) ; 4,35(\mathrm{~d}$, $\left.J_{1,2 \beta}=J_{5,4 \beta}=4,7 \mathrm{~Hz}, \mathrm{H}-1, \mathrm{H}-5\right) ; 5,16(\mathrm{~s}, \mathrm{H}-6, \mathrm{H}-7) ; 7,05$ (ddd, $J_{3, \mathrm{~F}}=$ $12,7 \mathrm{~Hz}, J_{3^{\prime}, 4}=8,1 \mathrm{~Hz}, J_{3^{\prime}, 5},=1,2 \mathrm{~Hz}, \mathrm{H}-3^{\prime}$ ); 7,13 (ddd, $J_{5,, 6}=8,1 \mathrm{~Hz}$, $J_{5^{\prime}, 4^{\prime}}=7,6 \mathrm{~Hz}, J_{5^{\prime}, 3^{\prime}}=1,2 \mathrm{~Hz}, \mathrm{H}-5$ '); 7,24-7,29 (m, $J_{4^{\prime}, 6^{\prime}}=1,8 \mathrm{~Hz}, \mathrm{H}-$ $4^{\prime}$ ), 7,39 (ddd, $J_{6^{\prime}, 5^{\prime}}=8,1 \mathrm{~Hz}, J_{6^{\prime}, \mathrm{F}^{\prime}}=8,1 \mathrm{~Hz}, J_{6^{\prime}, 4^{\prime}}=1,8 \mathrm{~Hz}, \mathrm{H}-6$ '). RMN ${ }^{13} \mathbf{C}\left(100 \mathrm{MHz}, \mathrm{CDCl}_{3}\right): 159,90$ (d, $J=243,7 \mathrm{~Hz}, \mathrm{C}-2$ ') $)$ 136,68 (d, $J=9,9 \mathrm{~Hz}, \mathrm{C}-1$ '); 129,00 (d, $J=8,9 \mathrm{~Hz}, \mathrm{C}-6$ '); 125,40 (d, $J=8,2$ Hz, C-4'); 123,81 (d, $J=3,0 \mathrm{~Hz}, \mathrm{C}-5$ '); 116,16 (d, $J=23,2 \mathrm{~Hz}, \mathrm{C}-$ 3'); 110,85 ( $\left.\mathrm{CMe}_{2}\right)$; 83,21 (C-6, C-7); 79,05 (C-1, C-5); 70,86 (C3); 39,05 (C-2, C-4); 25,76 (Me); 24,28 (Me). EM, $m / z$ (\%): 279, 10 $\left(\left[\mathrm{C}_{15} \mathrm{H}_{16} \mathrm{O}_{4} \mathrm{~F}\right]^{+?}, \mathrm{M}-15,100\right) ; 218,98$ (25); 201,02 (20), 173,07 (25), 123,02 (100), 43,00 (74).

3-butil-6,7-exo-isopropilidenodioxi-8-oxabiciclo[3.2.1]octan-3 $\alpha$-ol (15): A uma solução de magnésio $(0,245 \mathrm{~g}, 10,1 \mathrm{mmol})$ e alguns 
cristais de iodo, em THF anidro ( $3 \mathrm{~mL}$ ), adicionou-se $1 \mathrm{~mL}$ de uma solução de 1-bromobutano (1,38 g, 10,1 mmol, 2 mL de THF anidro), sob atmosfera de nitrogênio. A mistura foi mantida sob agitação magnética por $30 \mathrm{~min}$. Em seguida, adicionou-se o restante da solução de 1-bromobutano (1 mL), mantendo-se a agitação magnética por $2 \mathrm{~h}$. Logo após foi adicionada a solução do acetonídeo $6(0,2 \mathrm{~g}$, 1,01 mmol, $4 \mathrm{~mL}$ de THF anidro) em um intervalo de $20 \mathrm{~min}$. A agitação magnética foi mantida durante 17 horas. Adicionou-se em seguida $60 \mathrm{~mL}$ de solução saturada de $\mathrm{NH}_{4} \mathrm{Cl}$ e o produto foi extraído com acetato de etila $(3 \times 30 \mathrm{~mL})$. A fase orgânica foi lavada com solução saturada de $\mathrm{NaCl}(20 \mathrm{~mL})$, secada sobre $\mathrm{MgSO}_{4}$ anidro e concentrada em evaporador rotatório. O sólido obtido foi purificado por cromatografia em coluna de sílica-gel (hexano/AcOEt 3:1) fornecendo o álcool 15 (0,146 g, 0,57 mmol, 56\%). $\mathrm{T}_{\mathrm{f}}=200-202{ }^{\circ} \mathrm{C}$. IV $\left(\mathrm{KBr}, \mathrm{cm}^{-1}\right) \overline{\mathrm{v}}_{\max }: 3450,2950,2840,1450,1375,1260,1200$, $1150,1050,850,800,675 . \mathbf{R M N}^{1} \mathbf{H}\left(400 \mathrm{MHz}, \mathrm{CDCl}_{3}\right) \delta: 0,90(\mathrm{t}$, H-4'); 1,28 (m, H-2', H-3'); 1,33 (s, Me); 1,38 (m, H-1'); 1,49 (s, $\mathrm{Me}) ; 1,57(\mathrm{~s}, \mathrm{OH}) ; 1,61\left(\mathrm{~d}, J_{2 \alpha, 2 \beta}=J_{4 \alpha, 4 \beta}=14,6 \mathrm{~Hz}, \mathrm{H}-2 \alpha, \mathrm{H}-4 \alpha\right)$; $1,87\left(\mathrm{dd}, J_{2 \beta, 2 \alpha}=J_{4 \beta, 4 \alpha}=14,6 \mathrm{~Hz}, J_{2 \beta, 1} \stackrel{4 \alpha, 4 \beta}{=} J_{4 \beta, 5}=4,6 \mathrm{~Hz}, \mathrm{H}-2 \beta, \mathrm{H}-4 \beta\right)$; $4,23\left(\mathrm{~d}, J_{1,2 \beta}=J_{5,4 \beta}=4,6 \mathrm{~Hz}, \mathrm{H}-1, \mathrm{H}-5\right) ; 5,00$ (s, H-6, H-7). RMN ${ }^{13} \mathbf{C}$ (100 MHz, CDCl $)$ : 111,10 $\left(\mathrm{CMe}_{2}\right)$; 83,52 (C-6, C-7); 79,40 (C-1, C-5); 70,13 (C-3); 45,46 (C-2, C-4); 39,86 (C-1'); 26, 17 (Me); 24,59 (Me); 24,59 (C-2'); 22,96 (C-3'); 14,01 (C-4').

3-(2,4-dimetoxifenil)-6,7-exo-isopropilidenodioxi-8-oxabiciclo[3.2.1]octan-3 $\alpha$-ol (16): O álcool 16 foi preparado de acordo com o procedimento descrito para o composto 11, utilizando-se 1bromo-2,4-dimetoxibenzeno (2 $\mathrm{mmol})$, butil lítio $(2,52 \mathrm{mmol})$, acetonídeo 6 (0,5 mmol), obtendo-se um rendimento de $20 \%$ (0,033 g, $0,1 \mathrm{mmol}) \cdot \mathrm{T}_{\mathrm{f}}=152-153,5^{\circ} \mathrm{C} \cdot \mathbf{I V}\left(\mathrm{KBr}, \mathrm{cm}^{-1}\right) \overline{\mathrm{v}}_{\max }: 3400,2910,2900$, 1610, 1580, 1500, 1450, 1400, 1400, 1260, 1200, 1150, 1100, 1050, 850, 800. RMN ${ }^{1} \mathbf{H}\left(400 \mathrm{MHz}, \mathrm{CDCl}_{3}\right) \delta: 1,37$ (s, Me); 1,51 (s, Me); $1,51(\mathrm{~s}, \mathrm{OH}) ; 2,07\left(\mathrm{~d}, J_{2 \alpha, 2 \beta}=J_{4 \alpha, 4 \beta}=14,5 \mathrm{~Hz}, \mathrm{H}-2 \alpha, \mathrm{H}-4 \alpha\right) ; 2,36$ $\left(\mathrm{dd}, J_{2 \beta, 2 \alpha}=J_{4 \beta, 4 \alpha}=14,5 \mathrm{~Hz}, J_{2 \beta, 1}=J_{4 \beta, 5}=4,5 \mathrm{~Hz}, \mathrm{H}-2 \beta, \mathrm{H}-4 \beta\right) ; 3,79$ (s, OMe); 3,87 (s, OMe); 4,31 (d, $\left.J_{1,2 \beta}=J_{5,4 \beta}=4,5 \mathrm{~Hz}, \mathrm{H}-1, \mathrm{H}-5\right)$; 5,20 (s, H-6, H-7); 6,43 (dd, $J_{5^{\prime}, 6}=8,7 \mathrm{~Hz}, J_{5^{\prime}, 3},=2,5 \mathrm{~Hz}, \mathrm{H}-5$ '); 6,49 (d, $\left.J_{3,5},=2,5 \mathrm{~Hz}, \mathrm{H}-3^{\prime}\right)$; 7, 13 (d, $J_{6,5},=8,3 \mathrm{~Hz}, \mathrm{H}-6$ '). RMN ${ }^{13} \mathbf{C}(100$ $\mathrm{MHz}, \mathrm{CDCl}_{3}$ ): 156,85 (C-2'); 158,93 (C-4'); 126,24 (C-1'); 123,95 (C-6’); 102,95 (C-5'); 98,74 (C-3'); 108,17 ( $\left.\mathrm{CMe}_{2}\right)$; 82,56 (C-6, C7); 78,78 (C-1, C-5); 70,77 (C-3); 54,36 (OMe); 54,35 (OMe); 38,68 (C-2, C-4); 25,19 (Me); 23,65 (Me). EM, $\mathbf{m} / \mathbf{z}(\%)$ : 336,15 $\left(\left[\mathrm{C}_{18} \mathrm{H}_{24} \mathrm{O}_{6}\right]^{+\bullet}, 25\right) ; 318,14\left[\left(\mathrm{C}_{18} \mathrm{H}_{22} \mathrm{O}_{5}\right)^{+\bullet}, 15\right) ; 260,10(7), 231,00(22)$; 217,07 (23); 166,00 (10); 165,06 (100); 138,07 (10); 83,92 (12).

3-(2-fluorofenil)-6,7-exo-isopropilidenodioxi-8-oxabiciclo[3.2.1]oct-2eno (17), 3-(3-fluorofenil)-6,7-exo-isopropilidenodioxi-8-oxabiciclo[3.2.1] loct-2-eno (18) e 3-(3-clorofenil)-6,7-exo-isopropilidenodioxi-8-oxabiciclo[3.2.1]oct-2-eno (19): Os alquenos 17, 18 e 19 foram preparados de acordo com o procedimento descrito para os cloretos 9 e 10, e obtidos com rendimentos de 48, 80 e $31 \%$, respectivamente. As reações foram encerradas após $3 \mathrm{~h}$. Dados para 17: 0,135 g, 0,48 mmol. $\mathrm{T}_{\mathrm{f}}=94-95{ }^{\circ} \mathrm{C}$. IV $\left(\mathrm{KBr}, \mathrm{cm}^{-1}\right) \overline{\mathrm{v}}_{\text {max }}: 3040,3000,2950,1650$, $1500,1450,1250,1075,850,750 . \mathbf{R M N}^{1} \mathbf{H}\left(400 \mathrm{MHz}, \mathrm{CDCl}_{3}\right) \delta$ : 1,34 (s, Me); 1,54 (s, Me); 2,23 (d, $\left.J_{4 \alpha, 4 \beta}=17,8 \mathrm{~Hz}, \mathrm{H}-4 \alpha\right)$; 2,9 (ddd, $\left.J_{4 \beta, 4 \alpha}=17,8 \mathrm{~Hz}, J_{4 \beta, 5}=5,9 \mathrm{~Hz}, J_{4 \beta, 2}=0,9 \mathrm{~Hz}, \mathrm{H}-4 \beta\right) ; 4,48\left(\mathrm{~d}, J_{5,4 \beta}=5,9\right.$ $\mathrm{Hz}, \mathrm{H}-5) ; 4,56\left(\mathrm{~d}, J_{1,2}=4,9 \mathrm{~Hz}, \mathrm{H}-1\right) ; 4,67\left(\mathrm{~d}, J_{6,7}=5,6 \mathrm{H}-6\right) ; 4,77(\mathrm{~d}$, $\left.J_{7,6}=5,6 \mathrm{H}-7\right) ; 6,18\left(\mathrm{dd}, J_{2,1}=4,9 \mathrm{~Hz}, J_{2,4 \mathrm{~b}}=0,9 \mathrm{~Hz}, \mathrm{H}-2\right), 7,03(\mathrm{dd}$, $\left.J_{3^{\prime}, 4^{\prime}}=8,0 \mathrm{~Hz}, J_{3^{\prime}, \mathrm{F}^{\prime}}=11,5 \mathrm{~Hz}, \mathrm{H}-3^{\prime}\right) ; 7,09\left(\mathrm{dd}, J_{5^{\prime}, 4^{\prime}}=J_{5^{\prime}, 6^{\prime}}=7,7 \mathrm{~Hz}, \mathrm{H}-\right.$ 5'); 7,19-7,26 (m, H-4', H-6'). RMN ${ }^{13} \mathbf{C}\left(100 \mathrm{MHz}^{\prime}, \mathrm{CDCl}_{3}\right): 160,20$ (d, $J=274,0 \mathrm{~Hz}, \mathrm{C}-2$ '); 131,51 (C-3); 130,25 (C-2); 129,26 (d, $J=7,7$ Hz, C-4'); 127,51 (d, J = 5,5 Hz, C-6'); 127,37 (C-1'); 124,16 (d, $J=$ $\left.3,5 \mathrm{~Hz}, \mathrm{C}-5^{\prime}\right) ; 116,00$ (d, $\left.J=22,5 \mathrm{~Hz}, \mathrm{C}-3^{\prime}\right) ; 112,50\left(\mathrm{CMe}_{2}\right) ; 85,89 \mathrm{e}$ 85,69 (C-6, C-7); 79,34 (C-1); 76,78 (C-5); 31,75 (C-4); 26,25 (Me);
24,92 (Me). EM, $\boldsymbol{m} / \boldsymbol{z}(\%): 276,12\left(\left[\mathrm{C}_{16} \mathrm{H}_{17} \mathrm{O}_{3} \mathrm{~F}\right]^{+\bullet}, 44\right) ; 261,09$ $\left(\left[\mathrm{C}_{15} \mathrm{H}_{14} \mathrm{O}_{3} \mathrm{~F}\right]^{+*}, 16\right) ; 217,98$ (30); 190,00 (24); 189,07 (100); 175,02 (82); 146,03 (26); 133,00 (22), 109,05 (15); 83,92 (35); 48,97 (50). Dados para 18: $0,075 \mathrm{~g}, 0,27 \mathrm{mmol} . \mathrm{T}_{\mathrm{f}}=97-98{ }^{\circ} \mathrm{C}$. IV $\left(\mathrm{KBr}, \mathrm{cm}^{-1}\right)$ $\bar{v}_{\max }: 3000,2900,1610,1580,1375,1275,1200,1075,870,800 . \mathbf{R M N}$ ${ }^{1} \mathbf{H}\left(400 \mathrm{MHz}, \mathrm{CDCl}_{3}\right) \delta: 1,33(\mathrm{~s}, \mathrm{Me}) ; 1,54(\mathrm{~s}, \mathrm{Me}) ; 2,19\left(\mathrm{~d}, J_{4 \alpha, 4 \beta}=\right.$ $17,5 \mathrm{~Hz}, \mathrm{H}-4 \alpha$ ); 2,93 (ddd, $J_{4 \beta, 4 \alpha}=17,5 \mathrm{~Hz}, J_{4 \beta, 5}=6,0 \mathrm{~Hz}, J_{4 \beta, 2}=1,1$ $\mathrm{Hz}, \mathrm{H}-4 \beta) ; 4,54$ (d, $\left.J_{5,4 \beta}=6,0 \mathrm{~Hz}, \mathrm{H}-5\right) ; 4,59$ (d, $\left.J_{6,7}=6,0 \mathrm{~Hz}, \mathrm{H}-6\right)$; $4,61\left(\mathrm{~d}, J_{7,6}=6,0 \mathrm{H}-7\right) ; 4,72\left(\mathrm{~d}, J_{1,2}=5,6 \mathrm{H}-1\right) ; 6,33\left(\mathrm{dt}, J_{2,1}=5,6 \mathrm{~Hz}\right.$, $J_{2,4 \beta}=1,12 \mathrm{~Hz}, \mathrm{H}-2$ ), 6,97 (tdd, $J_{4^{\prime}, \mathrm{F}}=J_{4^{\prime}, 5^{\prime}}=8,0 \mathrm{~Hz}, J_{4^{\prime}, 2^{\prime}}=2,3 \mathrm{~Hz}, J_{4^{\prime}, 6^{\prime}}$ $\left.=0,8 \mathrm{~Hz}, \mathrm{H}-4^{\prime}\right) ; 7,03$ (dt, $\left.J_{2^{\prime}, \mathrm{F}}=10,5 \mathrm{~Hz}, J_{2^{\prime}, 4^{\prime}}=J_{2^{\prime}, 6^{\prime}}=2,3 \mathrm{~Hz}, \mathrm{H}-2^{\prime}\right)$; 7,19 (dt, $\left.J_{6,5},=8,0 \mathrm{~Hz}, J_{6^{\prime}, 4^{\prime}}=0,8 \mathrm{~Hz}, \mathrm{H}-6^{\prime}\right) ; 7,28$ (td, $J_{5^{\prime}, 4}=J_{5^{\prime}, 6^{\prime}}=8,0$ $\mathrm{Hz}, J_{5^{\prime}, \mathrm{F}}=6,0 \mathrm{~Hz}, \mathrm{H}-5$ '). RMN ${ }^{13} \mathbf{C}\left(100 \mathrm{MHz}, \mathrm{CDCl}_{3}\right)$ : 162,97 (d, $J_{C, F}$ $\left.=244,1 \mathrm{~Hz}, \mathrm{C}^{\prime} 3^{\prime}\right) ; 140,94$ (d, $\left.J_{C F}=7,4 \mathrm{~Hz}, \mathrm{C}-1^{\prime}\right) ; 133,60$ (C-3); 129,93 $\left(\mathrm{d}, J_{C, F}=8,4 \mathrm{~Hz}, \mathrm{C}-5\right.$ '); $124,25(\mathrm{C}-2) ; 120,38$ (d, $J_{C, F}=2,6 \mathrm{~Hz}, \mathrm{C}-6$ '); $114,75\left(\mathrm{~d}, J_{C, F}=21,1 \mathrm{~Hz}, \mathrm{C}-4\right.$ '); 112,38 $\left(\mathrm{CMe}_{2}\right) ; 111,76\left(\mathrm{~d}, J_{C, F}=22,1\right.$ Hz, C-2'); 85,64 (C-1); 79,10 (C-5), 76,70 (C-6 e C-7); 30,41 (C-4); 26,22 (Me); 24,88 (Me). EM, $\boldsymbol{m} / z(\%): 294,09\left(\left[\mathrm{C}_{16} \mathrm{H}_{21} \mathrm{O}_{3} \mathrm{~F}+\mathrm{NH}_{4}\right]^{+*}\right.$, 12); 277,12 ([C $\left.\left.\mathrm{C}_{16} \mathrm{H}_{18} \mathrm{O}_{3} \mathrm{~F}\right]^{+}, 15\right) ; 218,98$ (100); 175,01 (45); 146,02 (12). Dados para 19: 0,055g, 0,19 mmol. $\mathrm{T}_{\mathrm{f}}=93-94,2{ }^{\circ} \mathrm{C}$. IV $(\mathrm{KBr}$, $\left.\mathrm{cm}^{-1}\right) \bar{v}_{\text {max }}: 3000,2900,1600,1550,1260,1200,1100,850,760 . \mathbf{R M N}$ ${ }^{1} \mathbf{H}\left(400 \mathrm{MHz}, \mathrm{CDCl}_{3}\right) \delta: 1,35(\mathrm{~s}, \mathrm{Me}) ; 1,56(\mathrm{~s}, \mathrm{Me}) ; 2,21\left(\mathrm{~d}, J_{4 \alpha, 4 \mathrm{~s}}=\right.$ $16,6 \mathrm{~Hz}, \mathrm{H}-4 \alpha) ; 2,95$ (ddd, $J_{4 \beta, 4 \alpha}=16,6 \mathrm{~Hz}, J_{4 \beta, 5}=6,0 \mathrm{~Hz}, J_{4 \beta, 2}=0,9$ $\mathrm{Hz}, \mathrm{H}-4 \beta) ; 4,55$ (d, $\left.J_{5.4 \mathrm{\beta}}=6,0 \mathrm{~Hz}, \mathrm{H}-5\right) ; 4,61$ (d, $\left.J_{7.6}=6,0 \mathrm{~Hz}, \mathrm{H}-7\right)$; $4,63\left(\mathrm{~d}, J_{6,7}=6,0 \mathrm{H}-6\right) ; 4,73\left(\mathrm{~d}, J_{1,2}=5,6 \mathrm{~Hz}, \mathrm{H}-1\right) ; 6,33\left(\mathrm{dt}, J_{2,1}=5,6\right.$ $\left.\mathrm{Hz}, J_{2,4 \beta}=0,9 \mathrm{~Hz}, \mathrm{H}-2\right), 7,23-7,28$ (m, H-4', H-5', H-6'); 7,34 (s, H$\left.2^{\prime}\right)$. $\mathbf{R M N}{ }^{13} \mathbf{C}\left(100 \mathrm{MHz}, \mathrm{CDCl}_{3}\right)$ : 140,56 (C-3); 134,55 (C-1'); 133,58 (C-3'); 129,69 (C-5'); 127,90 (C-6'); 125,08 (C-4'); 124,45 (C-2); 122,91 (C-2'); 112,43 ( $\left.\mathrm{CMe}_{2}\right) ; 85,77$ (C-1); 79, 12 (C-5), 77,32 (C-6 e C-7); 30,44 (C-4); 26,24 (Me); 24,94 (Me). EM, m/z (\%): 292 $\left(\left[\mathrm{C}_{16} \mathrm{H}_{17} \mathrm{O}_{3} \mathrm{Cl}\right]^{+?}, 3\right) ; 277\left(\left[\mathrm{C}_{15} \mathrm{H}_{14} \mathrm{O}_{3} \mathrm{Cl}\right]^{+}, 5\right) ; 234$ (15); 205 (75); 191 (55); 153 (30); 141 (35); 43 (100).

1-(3-fluorofenil)butano-1,4-diol (27): IV $\left(\mathrm{KBr}, \mathrm{cm}^{-1}\right) \overline{\mathrm{v}}_{\max }: 3350,2930$, 2850, 1620, 1575, 1500, 1450, 1250, 1100, 1050, 850, 760, 680. RMN ${ }^{1} \mathbf{H}\left(400 \mathrm{MHz}, \mathrm{CDCl}_{3}\right) \delta: 1,60-1,70(\mathrm{~m}, \mathrm{H}-3) ; 1,77-1,89(\mathrm{~m}, \mathrm{H}-2, \mathrm{OH})$; 3,62-3,72 (m, H-4); 4,71 (dd, $J_{l}=7,4 \mathrm{~Hz}, J_{2}=4,9 \mathrm{~Hz}, \mathrm{H}-1$ ); 6,95 (tdd, $\left.J_{4^{\prime}, F}=J_{4^{\prime}, 5}=8,0 \mathrm{~Hz}, J_{4^{\prime}, 2^{\prime}}=2,3 \mathrm{~Hz} ; J_{4^{\prime}, 6^{\prime}}=0,8 \mathrm{~Hz}, \mathrm{H}-4^{\prime}\right) ; 7,09$ (m, H-2', H-6'); 7,28 (td, $\left.J_{5^{\prime}, 4^{\prime}}=J_{5^{\prime}, 6^{\prime}}=8,0 \mathrm{~Hz}, J_{5^{\prime}, F}=6,0 \mathrm{~Hz} ; \mathrm{H}^{\prime} 5^{\prime}\right)$. RMN ${ }^{13} \mathbf{C}$ $\left(100 \mathrm{MHz}, \mathrm{CDCl}_{3}\right): 162,43\left(\mathrm{~d}, J_{\mathrm{C}, \mathrm{F}}=244,4 \mathrm{~Hz}, \mathrm{C}-3^{\prime}\right) ; 147,46\left(\mathrm{~d}, J_{\mathrm{C}, \mathrm{F}}=\right.$ $\left.6,7 \mathrm{~Hz}, \mathrm{C}^{-1}{ }^{\prime}\right) ; 129,86\left(\mathrm{~d}, J_{\mathrm{C} . \mathrm{F}}=8,3 \mathrm{~Hz}, \mathrm{C}-5\right.$ '); $121,32\left(\mathrm{~d}, J_{\mathrm{C} . \mathrm{F}}=2,5 \mathrm{~Hz}\right.$, C-6'); 114,14 (d, $\left.J_{\mathrm{C}, \mathrm{F}}=21,1 \mathrm{~Hz}, \mathrm{C}-4^{\prime}\right) ; 112,67$ (d, $J_{\mathrm{C}, \mathrm{F}}=21,5 \mathrm{~Hz}, \mathrm{C}-$ 2'); 73,58 (C-1); 62,71 (C-4); 35,88 (C-2); 28,88 (C-3).

1-(3-fluorofenil)tetraidrofurano (28): IV $\left(\mathrm{KBr}, \mathrm{cm}^{-1}\right) \overline{\mathrm{v}}_{\text {max }}: 2953$, 2850, 1616, 1591, 1489, 1447, 1274, 1138, 1058, 872, 788, 694.

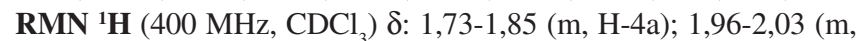
H-4b, H-3a); 2,28-2,36 (m, H-3b); 3,93 (ddd, $J_{1}=15,0$ Hz, $J_{2}=8,2$ $\left.\mathrm{Hz}, J_{3}=1,4 \mathrm{~Hz}, \mathrm{H}-5 \mathrm{a}\right) ; 4,08\left(\mathrm{ddd}, J_{1}=15,0 \mathrm{~Hz}, J_{2}=8,2 \mathrm{~Hz} ; J_{3}=1,4\right.$ $\mathrm{Hz}, \mathrm{H}-5 \mathrm{~b}) ; 4,88$ (t, $J=7,1 \mathrm{~Hz}, \mathrm{H}-1) ; 6,93$ (tdd, $J_{4^{\prime}, F}=J_{4^{\prime}, 5}=8,0 \mathrm{~Hz}$, $\left.J_{4^{\prime}, 2^{\prime}}=2,3 \mathrm{~Hz} ; J_{4^{\prime}, 6^{\prime}}=0,8 \mathrm{~Hz}, \mathrm{H}-4^{\prime}\right) ; 7,05\left(\mathrm{dt}, J_{2^{\prime}, F}=10,5 \mathrm{~Hz}, J_{2^{\prime}, 4^{\prime}}=J_{2^{\prime}, 6^{\prime}}\right.$ $=2,3 \mathrm{~Hz} ; \mathrm{H}-2^{\prime}$ ); 7,08 (d, $\left.J_{6,5},=8,0 \mathrm{~Hz}, \mathrm{H}-6^{\prime}\right) ; 7,27\left(\mathrm{td}, J_{5^{\prime},{ }^{\prime}}=J_{5^{\prime}, 6^{\prime}}=\right.$ $\left.8,0 \mathrm{~Hz}, J_{5, F}=6,0 \mathrm{~Hz}, \mathrm{H}-5^{\prime}\right)$. RMN ${ }^{13} \mathbf{C}\left(100 \mathrm{MHz} \mathrm{CDCl}_{3}\right): 165,92$ $\left(\mathrm{d}, J_{\mathrm{C}, \mathrm{F}}=243,8 \mathrm{~Hz}, \mathrm{C}^{-3} 3^{\prime}\right) ; 149,36\left(\mathrm{~d}, J_{\mathrm{C}, \mathrm{F}}=6,8 \mathrm{~Hz}, \mathrm{C}-1^{\prime}\right) ; 132,72(\mathrm{~d}$, $\left.J_{\mathrm{C} . \mathrm{F}}=7,9 \mathrm{~Hz}, \mathrm{C}-5^{\prime}\right) ; 124,09\left(\mathrm{~d}, J_{\mathrm{C} . \mathrm{F}}=2,8 \mathrm{~Hz}, \mathrm{C}-6\right.$ '); 116,81 (d, $J_{\mathrm{C} . \mathrm{F}}=$ $21,2 \mathrm{~Hz}, \mathrm{C}-4^{\prime}$ ); 115,42 (d, $J_{\mathrm{C}, \mathrm{F}}=22,1 \mathrm{~Hz}, \mathrm{C}^{2}$ ') ; 82,93 (C-2); 71,72 (C-5); 39,32 (C-3); 28,87 (C-4).

\section{Ensaios biológicos}

Para avaliação da atividade herbicida dos compostos 6, 7, 8, 9, $11,12,13,14,17$ e 19 foram realizados ensaios utilizando-se o Sorghum bicolor L. como planta indicadora, de acordo com a metodo- 
logia descrita na literatura ${ }^{17}$. A solução estoque foi preparada dissolvendo-se $5 \mathrm{mg}$ de cada composto em $20 \mu \mathrm{L}$ de pentan-3-ona e $60 \mu \mathrm{L}$ de xileno. A essa solução foram adicionadas duas gotas do surfactante Tween 40, sendo o volume final da solução completado com água destilada para $100 \mathrm{~mL}$. Uma solução de mesma composição, porém sem o composto a ser avaliado, foi utilizada como tratamento controle. O experimento para avaliação da fitotoxicidade sobre o desenvolvimento do sistema radicular e da parte aérea de $S$. bicolor foi conduzido em potes plásticos, aos quais se adicionou uma mistura de areia lavada e a solução do composto a ser avaliado. O delineamento experimental foi inteiramente casualizado com cinco repetições. Cada composto foi avaliado na concentração de 5,5 ppm. Em cada pote foram semeadas cinco sementes da espécie indicadora a 1 $\mathrm{cm}$ de profundidade, sendo os mesmos mantidos em sala de crescimento sob luminosidade e temperatura controladas (fotoperíodo de $12 \mathrm{~h}$, temperatura $25 \pm 3{ }^{\circ} \mathrm{C}$, radiação fotossinteticamente ativa 300 $\mu \mathrm{mol} \mathrm{m} \mathrm{m}^{-2} \mathrm{~s}^{-1}$ ), irrigados três vezes ao dia com água destilada, e três vezes por semana com solução nutritiva comercial. No vigésimo dia após a semeadura foram feitas avaliações visuais seguindo a escala da ALAM (Associação Latino-Americana de Malezas), atribuindose valores de zero (ausência de fitotoxicidade) a 100 (morte total da planta). O ensaio para avaliação do efeito dos compostos sobre o acúmulo de biomassa seca do sistema radicular e da parte aérea de $S$. bicolor foi realizado sob as mesmas condições descritas acima, porém utilizando-se solo como substrato. Todos os dados obtidos foram analisados estatisticamente, e para comparação de médias utilizou-se o teste de Tukey a $5 \%$ de probabilidade ${ }^{18}$.

\section{RESULTADOS E DISCUSSÃO}

\section{Síntese}

Álcoois e alquenos derivados do 8-oxabiciclo[3.2.1]oct-6-en-3ona (1) foram preparados de acordo com a metodologia apresentada no Esquema 1. Em trabalho anterior ${ }^{10}$ já havia sido relatada a síntese dos álcoois 12, 13 e 14 dos quais apenas 14 resultou no alqueno

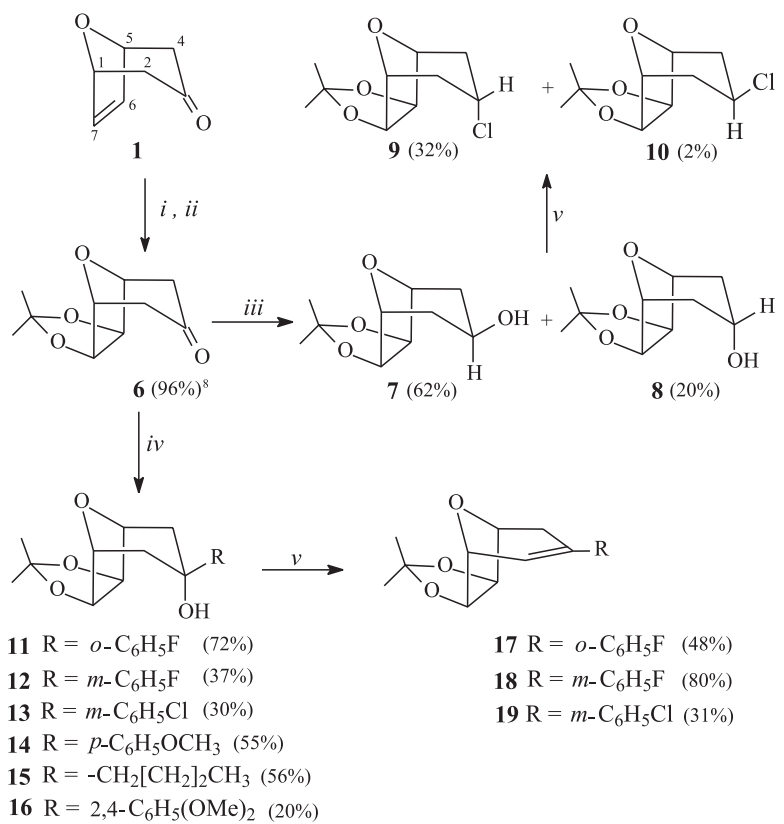

Esquema 1. i) $\mathrm{OsO}_{4}, \mathrm{H}_{2} \mathrm{O}_{2}$, acetona, Et $\mathrm{O}_{2} \mathrm{O}, 3$ dias, $25^{\circ} \mathrm{C}$; ii) acetona, PTSA, $\mathrm{CuSO}_{4}, 7$ dias, $25^{\circ} \mathrm{C}$; iii) $\mathrm{NaBH}_{4}, \mathrm{MeOH}, 5 \mathrm{~h}, 25 \rightarrow 40^{\circ} \mathrm{C}, 2 \mathrm{~h}$; iv) $\mathrm{BuLi}$, $\mathrm{THF},-78^{\circ} \mathrm{C}, 5,5 \mathrm{~h}(11), 26 \mathrm{~h}\left(14\right.$ e 16), $\mathrm{H}_{3} \mathrm{O}^{+}$; 12, 13 e 15: a) $\mathrm{ArMgBr}, \mathrm{THF}$, $24 \mathrm{~h}$, b) $\mathrm{NH}_{4} \mathrm{Cl}$ (aq.); v) $\mathrm{SOCl}_{2}$, Piridina, $25^{\circ} \mathrm{C}, 3 \mathrm{~h}(17,18,19)$; $7 \mathrm{~h}(\mathbf{9}, 10)$. correspondente ( $84 \%$ ), que causou $45 \%$ de inibição sobre a raiz de S. bicolor. Naquele estudo, cujo propósito foi a síntese e avaliação da fitotoxicidade de alquenos, não foram realizados ensaios com os álcoois precursores. Em outro estudo paralelo de investigação do potencial fitotóxico de derivados do 2,4-dimetil-8-oxabiciclo [3.2.1] oct-6-en-3-ona (20) sobre S. bicolor realizou-se o "screening" aleatório utilizando-se os álcoois 21, 22 e 23 e os alquenos 24, 25 e 26 (Figura 3), na concentração de 6,6 $\mu \mathrm{g} \mathrm{g}^{-1}$. Neste caso, os álcoois 21, 22 e 23 causaram $100 \%$ de inibição, enquanto os alquenos $\mathbf{2 4}$, $\mathbf{2 5}$ e $\mathbf{2 6}$ causaram 15, 32 e 37\%, respectivamente 9.

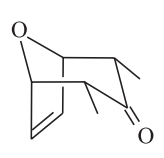

20

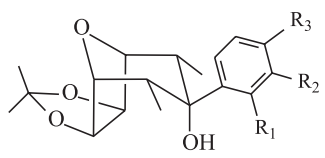

$21 \mathrm{R}_{1}=\mathrm{R}_{2}=\mathrm{H}, \mathrm{R}_{3}=\mathrm{F}$

$22 \mathrm{R}_{1}=\mathrm{R}_{2}=\mathrm{H}, \mathrm{R}_{3}=\mathrm{Me}$

$23 \mathrm{R}_{1}=\mathrm{R}_{2}=\mathrm{H}, \mathrm{R}_{3}=\mathrm{Cl}$

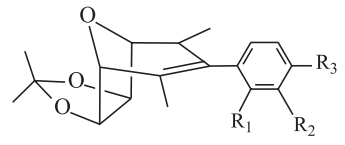

$24 \mathrm{R}_{1}=\mathrm{R}_{2}=\mathrm{H}, \mathrm{R}_{3}=\mathrm{F}$

$25 \mathrm{R}_{1}=\mathrm{R}_{2}=\mathrm{H}, \mathrm{R}_{3}=\mathrm{Me}$

$26 \mathrm{R}_{1}=\mathrm{R}_{2}=\mathrm{H}, \mathrm{R}_{3}=\mathrm{Cl}$
Figura 3. Álcoois e alquenos derivados do 2,4-dimetil-8-oxabiciclo [3.2.1] oct-6-en-3-ona (20)

Os resultados obtidos com os compostos 21, 22 e 23 motivaramnos, então, a preparar novamente os álcoois 12, 13 e 14 objetivandose avaliar a atividade fitotóxica dos mesmos.

Os cátions oxialílicos são de grande utilidade sintética por representarem intermediários no processo de síntese de diversos produtos naturais e estruturas correlacionadas; particularmente, a reação de cicloadição $[4+3]$ entre um cátion oxialílico, gerado in situ a partir de $\alpha$-halocetonas, e dienos gera cicloeptenos funcionalizados em diferentes posições ${ }^{12}$. A metodologia proposta iniciou-se com o preparo da 1,1,3,3-tetrabromopropanona, que foi obtida com rendimento de $88 \%$, conforme procedimento descrito na literatura ${ }^{19}$. O tratamento da 1,1,3,3-tetrabromopropanona com amálgama Zn/Ag, em THF, resultou no cátion oxialílico correspondente, cuja reação com furano forneceu uma mistura de 2,4-dibromo-8-oxabiciclo[3.2.1]oct-6-en3-ona (cicloadutos dibromados, não isolados). A redução dessa mistura com amálgama $\mathrm{Zn} / \mathrm{Cu}$, em presença de metanol, resultou no oxabiciclo 1. Em seguida, foi feita a oxidação de $\mathbf{1}$ com peróxido de hidrogênio, catalisada com tetróxido de ósmio. Essa reação resultou no diol correspondente, o qual foi convertido no acetonídeo $\mathbf{6}$, sem purificação prévia, mediante reação com acetona em presença de ácido $p$-toluenossulfônico e sulfato de cobre anidro.

Para a conversão do acetonídeo $\mathbf{6}$ nos álcoois derivados foram empregadas metodologias distintas. $\mathrm{O}$ tratamento de $\mathbf{6}$ com boroidreto de sódio, em metanol, forneceu os álcoois 7 (isômero exo, 62\%) e 8 (isômero endo, 20\%). A predominância relativa do isômero exo sobre o endo é decorrente da maior facilidade de ataque do agente redutor à face endo da molécula. Estudos conformacionais ${ }^{20}$ mostraram que o anel de seis membros nestes sistemas bicíclicos não adota verdadeiramente uma conformação em cadeira, pois a região do grupo carbonila encontra-se bem próxima ao plano das ligações $\mathrm{C} 1-\mathrm{C} 2$ e C3-C4. As absorções em 3350 e $3450 \mathrm{~cm}^{-1}$, observadas no espectro no infravermelho, identificaram o grupo hidroxila nas estruturas de 7 e 8, respectivamente. A multiplicidade dos sinais observada nos espectros de RMN ${ }^{1} \mathrm{H}$ para os hidrogênios metilênicos $\mathrm{H} 2$ e H4 é, notadamente, determinada pela posição relativa de ambos com o hidrogênio H3. Por exemplo, o duplo duplo dupleto em $\delta$ 1,67, atribuído aos hidrogênios metilênicos $\mathrm{H} 2 \beta$ e H4 $\beta$ no espectro de 8 , mostrou claramente o acoplamento geminal $\left(J_{2 \beta, 2 \alpha}=J_{4 \beta, 4 \alpha}=15,2 \mathrm{~Hz}\right)$ e o desdobramento do dupleto pelo acoplamento com os hidrogênios vicinais $\mathrm{H} 1, \mathrm{H} 3$ e $\mathrm{H} 5\left(J_{2 \beta, 3}=J_{4 \beta, 3}=1,3 \mathrm{~Hz}, J_{2 \beta, 1}=J_{4 \beta, 5}=0,9 \mathrm{~Hz}\right)$. Embora os hidrogênios metilênicos H2 $\beta$ e H4 $\beta$ de 7 também apresentem a mesma multiplicidade que foi observada em $\mathbf{8}$, foi possível 
estabelecer inequivocamente a estereoquímica do carbono $\mathrm{C} 3 \mathrm{em}$ ambos os casos, uma vez que o ângulo diedro $(\phi)$ entre H2 $\beta$ e H3 é próximo a $180^{\circ} \mathrm{em} 7$, e se aproxima de $60^{\circ}$ no caso de $\mathbf{8}$. Em função dessa disposição espacial, o álcool 7 foi identificado mediante a interpretação do sinal em $\delta=1,61$, que apresentou duas constantes de acoplamento de valor superior a $10 \mathrm{~Hz}\left(J_{2 \beta, 2 \alpha}=J_{4 \beta, 4 \alpha}=12,8 \mathrm{~Hz}, J_{2 \beta, 3}\right.$ $=J_{4 \beta, 3}=11,9 \mathrm{~Hz}, J_{2 \beta, 1}=J_{4 \beta, 1}=4,1 \mathrm{~Hz}$ ), já que o sinal em $\delta=1,67$ somente apresentou uma constante de acoplamento com valor superior a $10 \mathrm{~Hz}$.

Os álcoois $11(72 \%)$ e 16 (20\%) foram obtidos empregando-se a metodologia envolvendo aril lítio, preparado in situ a partir de butil lítio e brometo de arila ${ }^{9}$. O grupo hidroxila foi confirmado pela análise dos espectros no infravermelho obtidos para os álcoois 11 e 16, que apresentaram absorções em 3420 e $3400 \mathrm{~cm}^{-1}$, respectivamente. Os espectros de RMN ${ }^{1} \mathrm{H}$ dos álcoois oriundos do acetonídeo 6 apresentaram modificação aparente de deslocamento químico para os hidrogênios metilênicos $\mathrm{H} 2$ e H4, quando comparados com o espectro de 6. A presença da hidroxila confere maior blindagem a esses hidrogênios, o que acarreta diminuição do valor de deslocamento químico dos mesmos. A obtenção dos álcoois endo 11-16 está de acordo com os resultados obtidos anteriormente ${ }^{10}$ para aril álcoois derivados do acetonídeo 6. A estereoquímica do carbono C3 é determinada pela maior facilidade de ataque dos reagentes organometálicos (Grignard ${ }^{10}$ ou aril lítio) à face exo da molécula que, além de menos impedida, permite a coordenação do cátion metálico com o átomo de oxigênio em ponte. A fórmula molecular $\mathrm{C}_{18} \mathrm{H}_{24} \mathrm{O}_{6}$ do álcool $\mathbf{1 6}$ foi confirmada no espectro de massas, que apresentou o sinal do íon molecular em $\mathrm{m} / \mathrm{z}$ 336,15. No caso de 11 não se observou no espectro o sinal relativo ao íon molecular. O sinal de maior massa $(\mathrm{m} / \mathrm{z}$ 279,1 ) foi atribuído ao fragmento oriundo da perda de um grupo metil (M-15), que representa também o sinal de maior intensidade relativa (pico base).

A reação de $\mathbf{6}$ com brometo de 3-fluorofenilmagnésio forneceu o álcool 12 (37\%), conforme procedimento descrito anteriormen$\mathrm{te}^{10}$. Surpreendentemente isolou-se, além do produto desejado, o 1(3-fluorofenil)butano-1,4-diol (27), com rendimento de 16\% (Esquema 2), resultante do acoplamento do reagente de Grignard formado e o solvente, tetraidrofurano. Embora somente 27 tenha sido isolado, observou-se no decorrer da reação (monitoramento por cromatografia em camada delgada) a formação de uma mistura complexa, cujos compostos provavelmente resultem de desproporcionamento do reagente de Grignard, isomerização por via radicalar, dimerização ou ataque do reagente ao solvente ${ }^{21}$. A confirmação da estrutura proposta para o diol 27 deu-se, indiretamente, com a formação do éter 28 (obtido com rendimento de 93\%) a partir da desidratação do mesmo, sob aquecimento ${ }^{22}$.

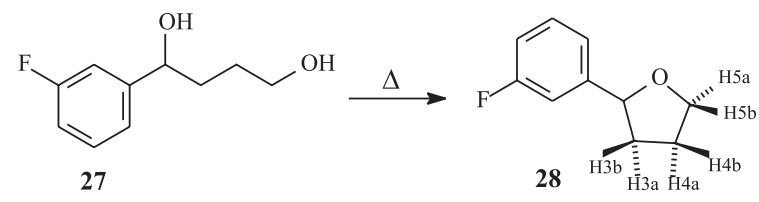

Esquema 2.

O álcool 15, resultante da adição do grupo butil à carbonila de $\mathbf{1}$, foi obtido com rendimento de $56 \%$ empregando-se a metodologia de Grignard e também obtido pela reação com butil lítio, porém com rendimento de $25 \%$. A absorção em $3450 \mathrm{~cm}^{-1}$, observada no espectro no infravermelho, identificou o grupo hidroxila na estrutura de 15. O espectro de $\mathrm{RMN}{ }^{13} \mathrm{C}$ apresentou sinais em $\delta 14,01, \delta 22,96, \delta$ 24,59 e $\delta 39,86$, que caracterizaram o grupo butil.

Das tentativas realizadas anteriormente para a desidratação dos álcoois 12, 13 e 14 com HCl, em acetona, somente a reação com 14 resultou no alqueno desejado ${ }^{10}$. Possivelmente os alquenos derivados de 12 e 13 não foram obtidos em decorrência da presença dos grupos $m$-fluorofenil e $m$-clorofenil, que diminuem a estabilidade relativa dos respectivos carbocátions, intermediários desta reação. Embora se tenha verificado, no caso da desidratação de 16, o consumo do material de partida durante a reação, não se observou a formação do alqueno desejado. A reação forneceu um produto de aspecto oleoso que não se revelou sobre luz ultravioleta $(\lambda=254 \mathrm{~nm})$ e com solução reveladora de $\mathrm{KMnO}_{4}$, sendo esses resultados impróprios com a identidade e propriedades de um alqueno. Para a desidratação dos álcoois 11, 12 e 13 utilizou-se a reação com cloreto de tionila, em presença de piridina ${ }^{23}$. As estruturas dos alquenos foram definidas mediante análises espectroscópicas, cujos resultados confirmaram as estruturas propostas e fórmulas moleculares, em todos os casos. Os espectros de RMN ${ }^{1} \mathrm{H}$ e ${ }^{13} \mathrm{C}$ dos alquenos 17, 18 e 19 apresentaram modificações aparentes, em relação aos espectros dos álcoois precursores. A perda de simetria molecular ocasionou aumento do número de sinais nos espectros e alterações no padrão de multiplicidade para alguns sinais no espectro de RMN ${ }^{1} \mathrm{H}$. Por exemplo, os hidrogênios equivalentes $\mathrm{H} 1$ e H5, observados como um dupleto em $\delta 4,35$ no espectro de 11, apresentaram-se como dupletos distintos no espectro de 17 ( $\delta$ 4,56 e 4,48, respectivamente). O acoplamento vicinal entre os hidrogênios H6 e H7, ausente nos álcoois, ocorreu nos alquenos, cujos espectros apresentaram dois dupletos ( $\delta$ 4,67 e 4,77, respectivamente) que foram atribuídos a esses hidrogênios. A reação de desidratação dos álcoois $\mathbf{7}$ e $\mathbf{8}$ com cloreto de tionila, em presença de piridina, forneceu os produtos clorados 9 e 10. Os espectros de $\mathrm{RMN}{ }^{13} \mathrm{C}$, que apresentaram um total de 7 sinais, juntamente com os espectros de $\mathrm{RMN}{ }^{1} \mathrm{H}$, evidenciaram a natureza simétrica dos produtos obtidos. O espectro de massas obtido para o cloreto 9 apresentou sinal do íon molecular em $\mathrm{m} / \mathrm{z}, 218$, que está de acordo com a fórmula molecular $\mathrm{C}_{10} \mathrm{H}_{15} \mathrm{O}_{3} \mathrm{Cl}$. A reação de desidratação de 7 e 8 foi realizada repetidas vezes e, do mesmo modo, somente forami obtidos como produtos os derivados clorados $\mathbf{9}$ e $\mathbf{1 0}$.

\section{Ensaios biológicos}

Dos compostos submetidos à avaliação da atividade herbicida sobre a parte aérea e o sistema radicular de S. bicolor, utilizando-se areia lavada como substrato (Tabela 1), apenas 8, 9 e 17 mostraramse inativos. Os demais compostos causaram inibição sobre a parte

Tabela 1. Fitotoxicidade dos compostos 6, 7, 8, 9, 11, 12, 13, 14, 17 e 19, na concentração de 5,5 ppm, sobre o desenvolvimento da parte aérea e do sistema radicular de Sorghum bicolor L., em areia lavada, 20 dias após a semeadura

\begin{tabular}{crr}
\hline Tratamentos & \multicolumn{2}{c}{$\%$ de Fitotoxicidade $*$} \\
& Parte aérea & Raiz \\
\hline Controle & $0,0 \mathrm{~d}$ & $0,0 \mathrm{e}$ \\
$\mathbf{6}$ & $75,6 \mathrm{a}$ & $93,6 \mathrm{a}$ \\
$\mathbf{7}$ & $15,2 \mathrm{c}$ & $0,0 \mathrm{~d}$ \\
$\mathbf{8}$ & $0,0 \mathrm{~d}$ & $0,0 \mathrm{e}$ \\
$\mathbf{9}$ & $0,0 \mathrm{~d}$ & $0,0 \mathrm{e}$ \\
$\mathbf{1 1}$ & $36,0 \mathrm{~b}$ & $76,0 \mathrm{~b}$ \\
$\mathbf{1 2}$ & $15,8 \mathrm{c}$ & $17,0 \mathrm{~d}$ \\
$\mathbf{1 3}$ & $2,4 \mathrm{~d}$ & $3,0 \mathrm{e}$ \\
$\mathbf{1 4}$ & $74,0 \mathrm{a}$ & $93,6 \mathrm{a}$ \\
$\mathbf{1 7}$ & $0,0 \mathrm{~d}$ & $0,0 \mathrm{e}$ \\
$\mathbf{1 9}$ & $31,6 \mathrm{~b}$ & $63,0 \mathrm{c}$ \\
\hline
\end{tabular}

*Médias seguidas por uma mesma letra não diferem entre si pelo Teste de Tukey a 5\% de probabilidade. 
aérea e raiz do sorgo, sendo a maior inibição observada sobre a raiz provocada pelo acetonídeo 6 (93,6\%) e pelo álcool 14 (93,6\%). Exceção ocorreu com o álcool 7, que somente causou inibição sobre o desenvolvimento da parte aérea $(15,2 \%)$. Os resultados obtidos nesse ensaio permitiram estabelecer a correlação estrutural preliminar dos derivados do 8-oxabiciclo[3.2.1] oct-6-en-3-ona (1) com a atividade herbicida. Ao se comparar os resultados do acetonídeo 6 com aqueles do álcool $\mathbf{8}$, produto da redução do composto $\mathbf{6}$, nota-se que a atividade herbicida sobre a parte aérea e raiz foi extinta, sendo o composto 8 inativo nessas condições. Esses resultados atribuem, inicialmente, o efeito de inibição à presença do grupo carbonila na molécula. Entretanto, o álcool 14 mostrou-se tão ativo quanto o acetonídeo 6, pois os resultados não diferem estatisticamente entre si. Portanto, a atividade fitotóxica não é dependente do grupo carbonila. Há de se considerar também a notável influência do grupo 4-metoxifenil, cujas propriedades fisico-químicas certamente contribuem para uma maior eficácia na inibição, em relação aos álcoois 11, 12 e 13.

A fitotoxicidade observada sobre a parte aérea e raiz com os álcoois 11 e 12 (Tabela 1) mostra que a presença do átomo de flúor em posição orto no anel benzênico confere significativa melhoria de atividade herbicida, em relação à substituição em meta. Considerando-se ainda uma mesma posição de substituição no anel benzênico (substituição em meta), observa-se que a atividade é dependente do tipo de halogênio, pois a inibição causada pelo álcool $\mathbf{1 3}$ foi inferior à de 12. A influência do padrão de substituição do anel aromático halogenado na atividade observada com os alquenos 17 e 19 mostrou-se contrária em relação aos álcoois precursores 11 e 13, respectivamente. Observa-se no caso do composto 19 que a presença do átomo de cloro em posição meta conferiu maior atividade, contrariamente ao observado com o álcool 13, que apresentou o menor percentual de inibição entre os aril álcoois substituídos. Considerando-se a substituição em posição orto pelo átomo de flúor, nota-se perda total de atividade do alqueno $\mathbf{1 7}$, em relação ao álcool 11, o qual apresentou expressiva taxa de inibição (76\%) sobre o sistema radicular de $S$. bicolor.

No ensaio em que se utilizou solo como substrato para desenvolvimento da planta indicadora buscou-se avaliar a fitotoxicidade através da resposta total da planta, em função do acúmulo de biomassa seca. Os resultados obtidos estão apresentados em percentagem de

Tabela 2. Efeito dos compostos 6, 7, 8, 9, 11, 12, 13, 14, 17 e 19 na concentração de 5,5 ppm, sobre o acúmulo de biomassa seca da parte aérea e do sistema radicular de Sorghum bicolor L., em solo, 20 dias após a semeadura

\begin{tabular}{clrlc}
\hline Tratamentos & \multicolumn{4}{c}{ Biomassa seca } \\
& \multicolumn{2}{c}{ Parte aérea } & \multicolumn{2}{c}{ Raiz } \\
& g/planta & \% inibição * & g/planta & $\%$ inibição * \\
\hline Controle & $0,1256 \mathrm{abc}$ & 0,0 & $0,1236 \mathrm{abc}$ & 0,0 \\
$\mathbf{6}$ & $0,0786 \mathrm{~cd}$ & 37,4 & $0,1154 \mathrm{abc}$ & 6,6 \\
$\mathbf{7}$ & $0,1340 \mathrm{ab}$ & $-6,7$ & $0,1352 \mathrm{ab}$ & $-9,0$ \\
$\mathbf{8}$ & $0,1162 \mathrm{abcd}$ & 7,5 & $0,1288 \mathrm{abc}$ & $-4,0$ \\
$\mathbf{9}$ & $0,1440 \mathrm{a}$ & $-14,6$ & $0,0974 \mathrm{abc}$ & 21,2 \\
$\mathbf{1 1}$ & $0,0940 \mathrm{bcd}$ & 25,2 & $0,0650 \mathrm{c}$ & 47,4 \\
$\mathbf{1 2}$ & $0,1200 \mathrm{abc}$ & 4,5 & $0,0816 \mathrm{bc}$ & 34,0 \\
$\mathbf{1 3}$ & $0,1254 \mathrm{abc}$ & 0,2 & $0,1074 \mathrm{abc}$ & 13,1 \\
$\mathbf{1 4}$ & $0,0710 \mathrm{~d}$ & 43,5 & $0,0686 \mathrm{bc}$ & 44,5 \\
$\mathbf{1 7}$ & $0,1200 \mathrm{abc}$ & 4,5 & $0,078 \mathrm{bc}$ & 36,9 \\
$\mathbf{1 9}$ & $0,952 \mathrm{bcd}$ & 24,2 & $0,1206 \mathrm{abc}$ & 2,4 \\
\hline
\end{tabular}

*Médias seguidas por uma mesma letra não diferem entre si pelo Teste de Tukey a 5\% de probabilidade. inibição do crescimento da raiz e parte aérea de $S$. bicolor em relação ao tratamento controle (Tabela 2). A baixa inibição causada pelo acetonídeo 6 sobre a raiz $(6,6 \%)$ e parte aérea $(37,4 \%)$, em relação ao ensaio realizado em areia lavada (93,6 e 75,6\%, respectivamente), evidenciou o grau de especificidade de um ensaio em função do tipo de resposta obtida. Não há incompatibilidade nesse tipo de resultado, uma vez que a diminuição de biomassa não necessariamente se correlaciona com a observação de fitotoxicidade. Pode-se obter, por exemplo, diminuição do diâmetro da raiz ou do número de raízes secundárias sem que ocorra diminuição no comprimento da raiz principal. Observa-se também que a atividade sobre a planta varia em função da parte considerada, pois um mesmo composto (8 e 9) pode causar estímulo ou inibição de crescimento em diferentes partes da planta.

\section{CONCLUSÃO}

Para avançar na investigação do potencial fitotóxico de derivados do 8-oxabiciclo[3.2.1] oct-6-en-3-ona (1) foram preparados novos álcoois e alquenos. Os álcoois foram obtidos com rendimentos de $20-72 \%$, em um total de três etapas, partindo-se da reação de cicloadição [4+3] entre furano e 1,1,3,3-tetrabromopropanona, e os alquenos obtidos com rendimentos de $31-80 \%$ pela desidratação dos álcoois correspondentes.

Os resultados obtidos com os compostos sintetizados mostraram uma variação de efeito em decorrência das condições de ensaio, tipo de substrato e parte da planta avaliada. No ensaio utilizando-se areia lavada como substrato para o desenvolvimento das plantas indicadoras os compostos causaram, em geral, maior inibição sobre o desenvolvimento da raiz, quando comparados com o resultado do ensaio em que se utilizou solo como substrato. Possivelmente, quando se utilizou solo como substrato parte do composto foi neutralizado pelas cargas dos colóides do solo, ocorrendo a sorção. Também para alguns dos compostos, a elevada porosidade do substrato areia lavada pode ter permitido a lixiviação desses compostos para a parte inferior dos potes. Esse fato pode ter reduzido a absorção desses compostos pelas raízes das plantas-teste e, conseqüentemente, seus efeitos danosos às mesmas.

\section{AGRADECIMENTOS}

À Coordenação de Aperfeiçoamento de Pessoal de Nível Superior (CAPES) pela bolsa de estudo (F. A. Ganen), ao Conselho Nacional de Desenvolvimento Cientifico e Tecnológico (CNPq) pelas bolsas de pesquisa (A. J. Demuner e L. C. A. Barbosa), à Fundação de Amparo à Pesquisa de Minas Gerais (FAPEMIG) e ao CNPq pelo apoio financeiro. Agradecemos o Prof. Dr. B. L. Hensen (México, UNAM) pelas sugestões apresentadas durante a discussão de resultados dos ensaios biológicos.

\section{REFERÊNCIAS}

1. Rodrigues, B. N.; Almeida, F. A.; Guia de Herbicidas, $4^{\mathrm{a}}$ ed., Edição dos Autores: Londrina, 1998.

2. Ragsdale, N. N.; Kearney, P. C.; Plimmer, J. R.; Eigth International Congress of Pesticide Chemistry, American Chemical Society: Washington, USA, 1995.

3. Cobb, A.; Herbicides and Plant Physiology, Chapman \& Hall: London, 1992.

4. Vargas, L.; Silva, A. A.; Borém, A.; Rezende, S. T.; Ferreira, F. A.; Sediyama, C. S.; Resistência de Plantas Daninhas a Herbicidas, Ed. UFV: Viçosa, 1999.

5. Barbosa, L. C. A.; Demuner, A. J.; Borges, E. E. L.; Mann, J.; J. Braz. Chem. Soc. 1997, 8, 19.

6. Barbosa, L. C. A.; Demuner, A. J.; Costa, A. V.; Borges, E. E. L.; Mann, J.; Quim. Nova 2000, 23, 461. 
7. Barbosa, L. C. A.; Maltha, C. R. A.; Borges, E. E. L.; Quim. Nova 2002, 25, 203.

8. Barbosa, L. C. A.; Demuner, A. J.; Maltha, C. R. A.; Silva, P. S.; Silva, A. A.; Quim. Nova 2003, 26, 655.

9. Costa, A. V.; Barbosa, L. C. A.; Demuner, A. J.; Silva, A. A.; J. Agric. Food Chem. 1999, 47, 4807.

10. Barbosa, L. C. A.; Conceição, G. J. A.; Demuner, A. J.; Silva, A. A.; Mann, J.; Piló-Veloso, D.; Aust. J. Chem. 1999, 52, 929.

11. Barbosa, L. C. A.; Maltha, C. R. A.; Demuner, A. J.; Filomeno, C. A.; Silva, A. A.; Quim. Nova 2004, 27, 241.

12. Demuner, A. J.; Barbosa, L. C. A.; Piló-Veloso, D.; Quim. Nova 1997, 20, 18.

13. Elliot, M.; Farnham, A. W.; Janes, N. F.; Johnson, D. M.; Pulman, D. A.; Pestic. Sci. 1987, 18, 223.

14. Elliot, M.; Farnham, A. W.; Janes, N. F.; Johnson, D. M.; Pulman, D. A.; Pestic. Sci. 1987, 18, 229.
15. Elliot, M.; Farnham, A. W.; Janes, N. F.; Johnson, D. M.; Pulman, D. A.; Pestic. Sci. 1987, 18, 239.

16. Perrin, D. D.; Armarego, W. L.; Purification of Laboratory Chemicals, $3^{\text {rd }}$ ed., Butterworth-Heinemann Ltd.: Londres, 1988.

17. Barbosa, L. C. A.; Alvarenga, E. S.; Demuner, A. J.; Figueiredo, R.; Silva, A. A.; Pest. Manag. Sci. 2003, 59, 1043.

18. Gomes, F. P.; Curso de Estatística Experimental, 3a ed., Nobel: Piracicaba, 1990

19. Aschroft, M. R.; Hoffmann, H. M. R.; Org. Synth. 1978, 55, 17.

20. Hoffmann, H. M. R.; Angew. Chem., Int. Ed. 1973, 12, 819.

21. Walborski, H. M.; Acc. Chem. Res. 1990, 23, 286.

22. Ao se utilizar éter dietílico como solvente em algumas reações de Grignard (resultados não publicados), observou-se semelhantemente a formação dos compostos 1-(4-clorofenil)etanol e 1-(4-metoxifenil)etanol, cujas estruturas foram confirmadas pela análise dos dados espectroscópicos.

23. Berstein, S.; Allen, W. S. J.; Org. Chem. 1954, 77, 1028. 